\title{
Comparing 1st Pass Success Rate and Number of Attempts for Internal Jugular Vein Cannulation by USG Guided Approach: Short Axis versus Long Axis Technique
}

\author{
Shallu Chaudhary ${ }^{1}$, Ravikant Dogra ${ }^{2}$, Ramesh Kumar ${ }^{3}$, \\ Rajesh Kumar Verma ${ }^{4}$, Major Amit Atwal ${ }^{5}$
}

${ }^{1}$ Medical Officer (Anaesthesia), Health \& Family Welfare Department, Himachal Pradesh, India
${ }_{2,3,4}$ Associate Professor, Department of Anesthesiology, IGMC Shimla Himachal Pradesh, India
${ }^{5}$ Medical Officer, Military Hospital Jutogh Cantt, Shimla, Himachal Pradesh, India

Corresponding Author: Major Amit Atwal

\begin{abstract}
The study was carried out in 80 patients admitted in ICU and OT at IGMC Shimla. Patients were divided into 2 groups:- group A (short axis) and group $\mathrm{B}$ (long axis) of 40 patients each. Internal jugular vein cannulation was done under USG guidance using the two techniques. We were successfully able to cannulate all the patients. We obtained vascular access with higher first pass success and less number of needle passes using short axis approach compared to long axis.
\end{abstract}

Keywords: internal jugular vein cannulation, USG guided approach, short axis versus long axis technique, Central vein catheterization

\section{INTRODUCTION}

Central vein catheterization is an integral part of invasive monitoring and management in the modern era. It is the standard clinical method for monitoring central venous pressure, therapeutic interventions like securing vascular access for administration of vasoactive drugs or to initiate rapid fluid resuscitation. The internal jugular vein route for central venous access has been described as early as in 1966 by Hermosura $^{1}$ and coworkers. IJV cannulation using USG guidance has become a common practice as it provides improved safety profile $^{2}$. It has been found that USG guidance could improve the success rate, reduce the number of needle passes and decrease complications ${ }^{3}$.

The use of ultrasound converts a blind procedure into a procedure under vision, improving the success. Ultrasound imaging of the internal jugular vein may be oriented along either short axis or long axis. In the short axis approach, both artery and vein can be seen simultaneously and minimum probe adjustment is required. While in the long axis, the operator can visualize the entire length of the needle as it punctures the target vessel. Using the long axis view, information regarding the location of the carotid artery relative to the internal jugular vein may be lost.

\section{MATERIAL AND METHODS}

The proposed study was carried out in 80 patients admitted in ICU and operation theatres at IGMC Shimla. The patients with indication of CVP catheter insertion and giving consent themselves or by their relatives were included in the study. Patients were randomized into 2 groups based on a random allocation number table. Keeping upto $95 \%$ confidence levels and $80 \%$ power of study, sample size for each group was calculated to be 40. Group A -short axis USG guided approach, Group B- long axis 
USG guided approach. Patients with distorted neck anatomy, previous neck surgery, prior IJV cannulation, BMI $>30 /<18$ were excluded from the study.

Before cannulation, the baseline vitals of the patients were recorded. After ensuring adequate sedation and analgesia, cannulation of the right IJV was performed using Seldinger technique under USG guidance with either of the technique as per the group of the patient. While cannulation was being performed, an observer unskilled in ultrasound guidance who was unaware of the group allocation observed the procedure and recorded the following information:-

- First pass success

- Total number of needle passes (number of times needle was withdrawn and redirected).

\section{OBSERVATION AND RESULTS Comparison of first pass success:}

In both the groups, our success rate of IJV cannulation was $100 \%$ in all the patients. From both the groups, $48.8 \%$ (39) patients were cannulated in first attempt. In group A first attempt success was achieved in $72.5 \%$ (29) patients, while in group B first attempt success was achieved in $25 \%$ (10) patients, p value of 0.000 which was found to be statistically significant. Thus the first pass success rate was significantly higher in group A (short axis) comparing to the group B (long axis). This may be because in short axis view both the artery and vein are seen simultaneously on the screen and thus during cannulation, hand eye coordination becomes much easier enabling high first pass success rate in cannulation.

\section{Comparison of number of needle attempts for CVP insertion:}

In group $\mathrm{A}$, first attempt cannulation was successful in $72.5 \%$ (29) patients, while second attempt was needed in $27.5 \%$ (11) patients. No third attempt was needed in group A patients. The mean number of needle attempts was $1.27 \pm 0.45$

Whereas in group B, first attempt cannulation was successful in $25 \%$ (10) patients, second attempt in 50\% (20) and third attempt was needed in $25 \%(10)$ patients. Mean number of needle attempts was $2.00 \pm 0.72$. This difference was found to be statistically significant ( $p$ value $<0.0001$ ). Thus the mean number of needle passes was significantly lesser in the short axis group when compared to the long axis.

\section{DISCUSSION}

The number of needle passes was significantly lower and the $1^{\text {st }}$ pass success rate was significantly higher in the short axis view as compared to the long axis. This may be because in short axis view both the artery and vein are seen simultaneously on the screen and thus during cannulation hand eye coordination becomes much easier enabling high first pass success in cannulation. While long axis view needs more hand eye coordination as single vessel is seen and cannot be compared with the adjacent vessel. Some time is consumed to confirm whether the vessel is an artery or vein. Therefore the long axis approach becomes technically more difficult.

Our study is similar to the study done by Chittoodan $\mathrm{S}$ et $\mathrm{al}^{4}$. In their study, $98 \%$ of the patients of short axis group were cannulated in $1^{\text {st }}$ attempt, while $78 \%$ of the patients of long axis group were cannulated in $1^{\text {st }}$ attempt. Remaining patients required $2^{\text {nd }}$ attempt. No third attempt was required. While in our study, $3^{\text {rd }}$ attempt was required in $25 \%$ patients of long axis group.

Our study is also in accordance to the study done by Chaudhary $\mathrm{S}$ et $\mathrm{al}^{5}$ They found that in short axis approach 19 out of 25 patients $(76 \%)$ and in the long axis approach 23 out of 25 patients $(92 \%)$ were successfully cannulated in $1^{\text {st }}$ attempt. While $2^{\text {nd }}$ attempt was needed in cannulating 2 patients in long axis approach and 6 patients in short axis approach. These findings were comparable to our study.

Similarly a study was conducted by Tammam $\mathrm{TF}^{6}$ et al in 90 patients in which they compared USG guided short axis and long axis view with the landmark technique. This study revealed no major difference in 
short axis (1.13) and long axis (1.17) for mean number of attempts but for the landmark technique, number of attempts were $2.47(\mathrm{p}<0.001)$ much higher than USG guided approaches.

Our study is also in accordance with the study done by Blavias et $\mathrm{al}^{7}$.in their study, mean number of attempts in short axis was 4.18 and in long axis was 5.76 Though it was statistically not significant ( $p$ value 0.490 ) but was similar to our study in which also mean number of needle prick attempts were more in long axis compared to short axis view.

In a study conducted by Shreshta $\mathrm{S}$ et $\mathrm{al}^{8}, 37(90.2 \%)$ patients of the short axis group were cannulated in $1^{\text {st }}$ attempt while $4(9.8 \%)$ patients needed $2^{\text {nd }}$ attempt. In the long axis group, $38(92.7 \%)$ patients were cannulated in $1^{\text {st }}$ attempt, $3(7.3 \%)$ patients required $2^{\text {nd }}$ attempt. This difference was not statistically significant. While in our study, we found statistically significant difference between the 2 groups in terms of first pass success and total number of needle passes.

\section{CONCLUSION}

In our study, all the patients from both the groups were successfully cannulated. Thus the incidence of successful cannulation was $100 \%$. The first pass success rate was found to be significantly higher in short axis group compared to long axis group. Also the mean numbers of needle attempts were found to be less in short axis group compared to the long axis group. We obtained vascular access with higher first pass success and less number of needle attempts using short axis approach compared to long axis.

\section{Acknowledgement: None}

Conflict of Interest: None

Source of Funding: None Ethical Approval: Approved

\section{REFERENCES}

1. Hermosura B, Vanags L, Dickey MW. Measurement of pressure during intravenous therapy. JAMA.1966;195:181.

2. Peris A, Zagli G, Bonizzoli M, Cianchi G, Ciapetti M, Spina R, et al. Implantation of 3951 long term central venous catheters: Performances, risk analysis, and patient comfort after ultrasound. Anesth Analg. 2010;111:1194-201.

3. Randolph AG. Cook DJ, Gonzales CA. Pribble CG. Ultrasound guidance for placement of central venous catheters: A meta analysis of literature. Crit Care Med 1996:24:2053-8.

4. Chittoodan S, Breen D, Donnell BDO, Iohom G. Long versus short axis ultrasound guided approach for internal jugular vein cannulation: A prospective randomized controlled trial. Med Ultrason. 2011 Mar; 13(1): 21-5.

5. Chaudhari MS, Bharat Shah S, Vithal Kamat H. Ultrasound guided internal jugular vein cannulation with short and long axis approach- Technical ease and complications. Indian Journal of Clinical Anaesthesia, 2016;3(4):546-550.

6. Tammam TF, El-Shafey EM, Tammam HF. Ultrasound guided internal jugular vein access: Comparison between Short Axis and Long Axis Techniques. Saudi J Kidney Dis Transpl 2013;24(4):707-713.

7. Blaivas M, Brannam L, Fernandez E, Short axis versus Long axis approaches for teaching ultrasound guided vascular access on a new inanimate model. Acad Emerg Med. 2003 Dec;10(12):1307-11. doi: 10.1111/j.1553-2712.2003.tb00002.x.

8. Shrestha GS, Gurung A, Koirala S. Comparison between long and short term technique for ultrasound guided cannulation of internal jugular vein. Ann Card Anaesth 2016;19:288-92.

How to cite this article: Chaudhary S, Dogra R, Kumar $\mathrm{R}$ et.al. Comparing 1st pass success rate and number of attempts for internal jugular vein cannulation by USG guided approach: short axis versus long axis technique. International Journal of Science \& Healthcare Research. 2021; 6(3): 201-203. DOI: https://doi.org/ 10.52403/ijshr.20210734 